\title{
METRIC SUBREGULARITY AND CALMNESS FOR NONCONVEX GENERALIZED EQUATIONS IN BANACH SPACES*
}

\author{
XI YIN ZHENG ${ }^{\dagger}$ AND KUNG FU NG
}

\begin{abstract}
This paper concerns a generalized equation defined by a closed multifunction between Banach spaces, and we employ variational analysis techniques to provide sufficient and/or necessary conditions for a generalized equation to have the metric subregularity (i.e., local error bounds for the concerned multifunction) in general Banach spaces. Following the approach of Ioffe [Trans. Amer. Math. Soc., 251 (1979), pp. 61-69] who studied the numerical function case, our conditions are described in terms of coderivatives of the concerned multifunction at points outside the solution set. Motivated by the existing modulus representation and point-based criteria for the metric regularity, we establish the corresponding results for the metric subregularity. In the Asplund space case, sharper results are obtained.
\end{abstract}

Key words. metric subregularity, calmness, coderivative, normal cone, normal dual mapping

AMS subject classifications. 90C $31,90 \mathrm{C} 25,49 \mathrm{~J} 52$

DOI. $10.1137 / 090772174$

1. Introduction. In this paper we discuss metric subregularity of the generalized equation

$$
b \in F(x),
$$

where and throughout we assume that $F: X \rightrightarrows Y$ is a closed multifunction, $b \in Y$ is a given point, and $X, Y$ are Banach spaces. Following Dontchev and Rockafellar [6], we say that (GE) is metrically subregular at $a \in F^{-1}(b)$ if there exists $\tau \in[0,+\infty)$ such that

$$
d\left(x, F^{-1}(b)\right) \leq \tau d(b, F(x)) \forall x \text { close to } a .
$$

This property provides an estimate of how far a candidate $x$ (in a neighborhood of $a$ ) can be from the solution set of (GE). A multifunction $M: Y \rightrightarrows X$ is said to be calm at $(b, a) \in \operatorname{Gr}(M)$ if there exists $L \in(0,+\infty)$ such that

$$
d(x, M(b)) \leq L\|y-b\| \forall(y, x) \in \operatorname{Gr}(M) \text { close to }(b, a) .
$$

As observed by Henrion and Outrata [10], (GE) is metrically subregular at $a \in F^{-1}(b)$ if and only if $M=F^{-1}$ is calm at $(b, a)$. The metric subregularity and calmness have already been studied by many authors under various names (see $[3,6,7,8,9,10,14$, $15,19,20,21,22,28,29,33,34,35,36,37]$ and references therein). A well-known property (that is stronger than the metric subregularity) is the metric regularity of a multifunction that has also been studied extensively (see $[1,2,5,14,16,17,18,23$,

${ }^{*}$ Received by the editors September 25, 2009; accepted for publication (in revised form) January 28, 2010; published electronically April 14, 2010. This research was supported by an earmarked grant (GRF) from the Research Grant Council of Hong Kong and the National Natural Science Foundation of People's Republic of China (grant 10761012).

http://www.siam.org/journals/siopt/20-5/77217.html

$\dagger$ Department of Mathematics, Yunnan University, Kunming 650091, People's Republic of China (xyzheng@ynu.edu.cn).

${ }^{\ddagger}$ Department of Mathematics (and IMS), The Chinese University of Hong Kong, Shatin, New Territory, Hong Kong (kfng@math.cuhk.edu.hk). 
$24,25,26,27,32]$ and references therein). Explicitly, $F$ is metrically regular at $a$ for $b$ if there exists $\tau \in(0,+\infty)$ such that

$$
d\left(x, F^{-1}(y)\right) \leq \tau d(y, F(x)) \forall(x, y) \text { close to }(a, b) .
$$

Let $\operatorname{reg} F(a, b):=\inf \{\tau>0 \mid(1.2)$ holds $\}$. It is known (cf. [24, Theorem 4.5]) that if $X$ and $Y$ are Asplund spaces, then

$$
\operatorname{reg} F(a, b)=\inf _{\varepsilon>0} \sup \left\{\left\|\hat{D}^{*} F(x, y)^{-1}\right\| \mid x \in B(a, \varepsilon), y \in F(x) \cap B(b, \varepsilon)\right\},
$$

where $\hat{D}^{*} F(x, y)$ denotes the (Fréchet) coderivative of $F$ at $(x, y)$ and $\left\|\hat{D}^{*} F(x, y)^{-1}\right\|$ denotes the inner norm of $\hat{D}^{*} F(x, y)^{-1}$. (See section 2 for undefined terms and further notations.) The modulus of the metric subregularity of (GE) at $a \in F^{-1}(b)$ is denoted by subreg $F(a, b)$ and is defined by

$$
\operatorname{subreg} F(a, b):=\inf \{\tau \in(0, \infty) \mid(1.1) \text { holds }\} .
$$

The case $\operatorname{subreg} F(a, b)=\infty$ corresponds to that (GE) is not metrically subregular at $a$. (Here and throughout we adopt the convention that the infimum over the empty set is $\infty$.) Motivated by [24, Theorem 4.5] on the treatment regarding (1.3), one of our aims is to provide an estimate of the modulus of the metric subregularity. In particular, we prove that

$$
\operatorname{subreg} F(a, b) \leq \inf _{\varepsilon>0} \sup \left\{\left\|\hat{D}^{*} F(x, y)^{-1}\right\| \mid x \in B(a, \varepsilon) \backslash F^{-1}(b), y \in F(x) \cap B(b, \varepsilon)\right\} .
$$

Unlike (1.3), here we do not have an equality (see a counterexample after Theorem 3.1).

Let $f: X \rightarrow \mathbb{R} \cup\{+\infty\}$ be a proper lower semicontinuous function, and consider the special case that $Y=\mathbb{R}, b=0$, and

$$
F(x):=[f(x),+\infty) \quad \forall x \in X .
$$

In this case, (GE) reduces to the following inequality:

$$
f(x) \leq 0,
$$

while the metric subregularity (1.1) reduces to

$$
d(x, S) \leq \tau[f(x)]_{+} \forall x \text { close to } a,
$$

where $S=\{x \in X \mid f(x) \leq 0\}$ and $[f(x)]_{+}=\max \{f(x), 0\}$. Usually inequality (1.6) is said to have a local error bound at $a$ if there exists $\tau \in(0,+\infty)$ such that (1.7) holds. Error bound properties have important applications in sensitivity analysis and convergence analysis of mathematical programming. In recent years, error bound properties have been extensively studied (cf. $[3,7,12,20,21,22,28,30,34]$ and references therein). In particular, studies on error bounds have been well carried out in terms of subdifferentials; these studies are mainly carried out in two directions of approach. The first direction is described by the subdifferentials of $f$ at points inside the solution set $S$ and the normal cones of $S$. In this direction, it is known that if $f$ is convex, then inequality (1.6) has a local error bound at $a$ if and only if there exist $\tau, \delta \in(0,+\infty)$ such that

$$
N(S, x) \cap B_{X^{*}} \subset[0, \tau] \partial f(x) \quad \forall x \in S \cap B(a, \delta)
$$


(cf. $[3,11,12,20,35]$ ). The second direction is described only by the subdifferentials of $f$ at points outside the solution set $S$. In this direction, Ioffe [13] first studied error bound (under a different name) when $f$ is locally Lipschitz. His work has been followed by many others, and it is now well known that, for a proper semicontinuous function $f$ on a Banach space $X$ and positive constants $\kappa, \delta$, the following implication holds:

$$
d\left(0, \partial_{c} f(x)\right) \geq \kappa \forall x \in B(a, \delta) \backslash S \Longrightarrow(1.6) \text { has a local error bound at } a .
$$

In view of the facts that the coderivative for a multifunction is the counterpart of the subdifferential for a real-valued function and that

$$
D_{c}^{*} F(x, f(x))(1)=D_{c}^{*} F(x, f(x))\left(\Sigma_{\mathbb{R}}\right)=\partial_{c} f(x)
$$

(where $F$ is defined by (1.5)), it is natural to study the metric subregularity for (GE) in terms of coderivatives also along two directions of approach. In fact, in the first direction of approach, the authors [36] studied the metric subregularity of (GE) and proved that if $F$ is a convex closed multifunction between Banach spaces $X$ and $Y$, then (GE) is metrically subregular at $a$ if and only if there exist $\tau, \delta \in(0,+\infty)$ such that

$$
N\left(F^{-1}(b), x\right) \cap B_{X^{*}} \subset \tau D^{*} F(x, b)\left(B_{Y^{*}}\right) \quad \forall x \in F^{-1}(b) \cap B(a, \delta)
$$

(which extends the corresponding result for numerical functions). Recently, this result was further extended from the convex case to the subsmooth case in [37].

In the second direction of approach, Ledyaev and Zhu [19] established implicit multifunction theorems in terms of coderivative in the Fréchet smooth Banach space case. Inspired by [15] and [19], in terms of coderivatives at points outside the solution set, we consider the metric subregularity of (GE) in a general Banach space case. Note that (GE) is metrically subregular at $a$ if and only if the inequality $d(b, F(x)) \leq 0$ has a local error bound at $a$. Based on (1.8), it is a straightforward idea to provide some sufficient conditions of the metric subregularity for (GE) by finding some conditions which imply that the function $x \mapsto d(b, F(x))$ is lower semicontinuous and that

$$
\partial_{c} d(b, F(\cdot))(x) \subset D_{c}^{*} F(x, y)\left(\Sigma_{Y^{*}}\right) \quad \text { for some } y \in F(x) .
$$

Ledyaev and Zhu [19] established a relation similar to $(*)$ under the assumption that $X, Y$ are Banach spaces with Fréchet smooth Lipschitz bump functions and that $F$ is a closed upper semicontinuous multifunction. In this paper, we mainly consider the case when $F$ is a closed multifunction between two general Banach spaces. In this case, the function $x \mapsto d(b, F(x))$ is not even lower semicontinuous, and $\partial_{c} d(b, F(\cdot))$ cannot be described by $D_{c}^{*} F(\cdot, \cdot)$.

2. Preliminaries. Let $X^{*}$ denote the dual space of $X$. Let $B_{X}$ and $\Sigma_{X}$ denote the closed unit ball and unit sphere of $X$, respectively (similar notations for $X^{*}$ ). For $x \in X$ and $r>0$, we denote by $B(x, r)$ the open ball with center $a$ and radius $r$. For a closed subset $A$ of $X$ and a point $a$ in $A$, let $T_{c}(A, a)$ denote the Clarke tangent cone of $A$ at $a$, that is, $v \in T_{c}(A, a)$ if and only if, for each sequence $\left\{a_{n}\right\}$ in $A$ converging to $a$ and each sequence $\left\{t_{n}\right\}$ in $(0, \infty)$ decreasing to 0 , there exists a sequence $\left\{v_{n}\right\}$ in $X$ converging to $v$ such that $a_{n}+t_{n} v_{n} \in A \forall n$. We denote by $N_{c}(A, a)$ the Clarke normal cone of $A$ at $a$, that is,

$$
N_{c}(A, a):=\left\{x^{*} \in X^{*} \mid\left\langle x^{*}, h\right\rangle \leq 0 \quad \forall h \in T_{c}(A, a)\right\} .
$$


For $\varepsilon \geq 0$ and $a \in A$, the nonempty set

$$
\hat{N}_{\varepsilon}(A, a):=\left\{x^{*} \in X^{*} \mid \limsup _{\substack{A \\ \rightarrow a}} \frac{\left\langle x^{*}, x-a\right\rangle}{\|x-a\|} \leq \varepsilon\right\}
$$

is called the set of Fréchet $\varepsilon$-normals of $A$ at $a$. When $\varepsilon=0, \hat{N}_{\varepsilon}(A, a)$ is a convex cone and is called the Fréchet normal cone of $A$ at $a$; it will also be denoted by $\hat{N}(A, a)$. Let $N(A, a)$ denote the limiting (Mordukhovich) normal cone of $A$ at $a$, that is,

$$
N(A, a)=\limsup _{x \rightarrow a, \varepsilon \rightarrow 0^{+}} \hat{N}_{\varepsilon}(A, x)
$$

Thus, $x^{*} \in N(A, a)$ if and only if there exists a sequence $\left\{\left(x_{n}, \varepsilon_{n}, x_{n}^{*}\right)\right\}$ in $A \times R_{+} \times X^{*}$ such that $\left(x_{n}, \varepsilon_{n}\right) \rightarrow(a, 0), x_{n}^{*} \stackrel{w^{*}}{\rightarrow} x^{*}$ and $x_{n}^{*} \in \hat{N}_{\varepsilon_{n}}\left(A, x_{n}\right)$ for each $n$. It is known that

$$
\hat{N}(A, a) \subset N(A, a) \subset N_{c}(A, a)
$$

(cf. $[24,25])$. If $A$ is convex, then $N_{c}(A, a)=\hat{N}(A, a)$ is the normal cone in the sense of convex analysis; in this case,

$$
N_{c}(A, a)=\hat{N}(A, a)=\left\{x^{*} \in X^{*} \mid\left\langle x^{*}, x\right\rangle \leq\left\langle x^{*}, a\right\rangle \forall x \in A\right\} .
$$

Let $\phi: X \rightarrow \mathbb{R} \cup\{+\infty\}$ be a proper lower semicontinuous function,

$$
\operatorname{dom}(\phi):=\{x \in X \mid \phi(x)<+\infty\} \text { and } \operatorname{epi}(\phi):=\{(x, t) \in X \times \mathbb{R} \mid \phi(x) \leq t\} .
$$

For $x \in \operatorname{dom}(\phi)$ and $h \in X$, let $\phi^{\uparrow}(x, h)$ denote the generalized directional derivative introduced by Rockafellar (cf. [4]), that is,

$$
\phi^{\uparrow}(x, h):=\lim _{\varepsilon \downarrow 0} \limsup _{z \rightarrow \infty, t \downarrow 0} \inf _{w \in h+\varepsilon B_{X}} \frac{\phi(z+t w)-\phi(z)}{t},
$$

where the expression $z \stackrel{\phi}{\rightarrow} x$ means that $z \rightarrow x$ and $\phi(z) \rightarrow \phi(x)$. Let $\partial_{c} \phi(x)$ denote the Clarke-Rockafellar subdifferential of $\phi$ at $x$, that is,

$$
\partial_{c} \phi(x):=\left\{x^{*} \in X^{*} \mid\left\langle x^{*}, h\right\rangle \leq \phi^{\uparrow}(x, h) \quad \forall h \in X\right\} .
$$

Recall that the Fréchet subdifferential of $\phi$ at $x \in \operatorname{dom}(\phi)$ is defined as

$$
\hat{\partial} \phi(x):=\left\{x^{*} \in X^{*} \mid \liminf _{z \rightarrow x} \frac{\phi(z)-\phi(x)-\left\langle x^{*}, z-x\right\rangle}{\|z-x\|} \geq 0\right\} .
$$

It is well known (cf. [24]) that

$$
\hat{\partial} \phi(x) \subset \partial_{c} \phi(x) .
$$

When $\phi$ is convex, the Clarke-Rockafellar and Fréchet subdifferentials reduce to the one in the sense of convex analysis, that is,

$$
\partial_{c} \phi(x)=\hat{\partial} \phi(x)=\left\{x^{*} \in X^{*} \mid\left\langle x^{*}, y-x\right\rangle \leq \phi(y)-\phi(x) \quad \forall y \in X\right\} \quad \forall x \in \operatorname{dom}(\phi) .
$$


For a closed set $A$ in $X$, let $\delta_{A}$ denote the indicator function of $A$. It is known (see $[24,25])$ that

$$
N_{c}(A, a)=\partial_{c} \delta_{A}(a), \hat{N}(A, a)=\hat{\partial} \delta_{A}(a) \quad \forall a \in A,
$$

and

$$
\partial_{c} \phi(x)=\left\{x^{*} \in X^{*} \mid\left(x^{*},-1\right) \in N_{c}(\operatorname{epi}(\phi),(x, \phi(x)))\right\} \quad \forall x \in \operatorname{dom}(\phi) .
$$

Recall that a Banach space $X$ is called an Asplund space if every continuous convex function on $X$ is Fréchet differentiable at each point of a dense subset of $X$. It is well known (cf. [31]) that $X$ is an Asplund space if and only if every separable subspace of $X$ has a separable dual space. In particular, every reflexive Banach space is an Asplund space. In the case when $X$ is an Asplund space, Mordukhovich and Shao [25] proved that

$$
N_{c}(A, a)=\operatorname{cl}^{*}(\operatorname{co}(N(A, a))) \text { and } N(A, a)=\underset{x \rightarrow a}{\limsup } \hat{N}(A, x) .
$$

The following sum rule and fuzzy sum rule (cf. [4, 24, 25]) play important roles in variational analysis and are useful for our analysis.

Lemma 2.1. Let $X$ be a Banach space and $\phi_{1}, \phi_{2}: X \rightarrow \mathbb{R} \cup\{+\infty\}$ be proper lower semicontinuous functions. Let $x \in \operatorname{dom}\left(\phi_{1}\right) \cap \operatorname{dom}\left(\phi_{2}\right)$ be a local minimizer of $\phi_{1}+\phi_{2}$. Suppose that one of $\phi_{1}$ and $\phi_{2}$ is locally Lipschitz around $x$. Then

$$
0 \in \partial_{c} \phi_{1}(x)+\partial_{c} \phi_{2}(x) .
$$

If, in addition, $X$ is an Asplund space, then for any $\varepsilon>0$ there exist $x_{1}, x_{2} \in B(x, \varepsilon)$ such that $\left|\phi_{i}\left(x_{i}\right)-\phi_{i}(x)\right|<\varepsilon(i=1,2)$ and

$$
0 \in \hat{\partial} \phi_{1}\left(x_{1}\right)+\hat{\partial} \phi_{2}\left(x_{2}\right)+\varepsilon B_{X^{*}} .
$$

For a multifunction $F$ from $X$ to $Y$, the graph of $F$ is defined by

$$
\operatorname{Gr}(F):=\{(x, y) \in X \times Y \mid y \in F(x)\} .
$$

As usual, $F$ is said to be closed (respectively, convex) if $\operatorname{Gr}(F)$ is a closed (respectively, convex) subset of $X \times Y$. Let $(x, y) \in \operatorname{Gr}(F)$. The tangent derivative $D_{c} F(x, y)$ of $F$ at $(x, y)$ is defined by

$$
\operatorname{Gr}\left(D_{c} F(x, y)\right)=T_{c}(\operatorname{Gr}(F),(x, y)) .
$$

By virtue of different kinds of normal cones of $\operatorname{Gr}(F)$, one defines corresponding different kinds of coderivatives of $F$ as follows: For any $y^{*} \in Y^{*}$,

$$
\begin{aligned}
& \hat{D}^{*} F(x, y)\left(y^{*}\right):=\left\{x^{*} \in X^{*} \mid\left(x^{*},-y^{*}\right) \in \hat{N}(\operatorname{Gr}(F),(x, y))\right\}, \\
& D^{*} F(x, y)\left(y^{*}\right):=\left\{x^{*} \in X^{*} \mid\left(x^{*},-y^{*}\right) \in N(\operatorname{Gr}(F),(x, y))\right\},
\end{aligned}
$$

and

$$
D_{c}^{*} F(x, y)\left(y^{*}\right):=\left\{x^{*} \in X^{*} \mid\left(x^{*},-y^{*}\right) \in N_{c}(\operatorname{Gr}(F),(x, y))\right\} .
$$

The history of the coderivatives can be found in Mordukhovich's book [24].

Let $G: X \rightarrow 2^{Y}$ be a positively homogeneous multifunction (i.e., $\operatorname{Gr}(G)$ is a cone in $X \times Y)$. The norm of $G$ is defined by $\|G\|:=\sup \left\{\|y\| y \in G\left(B_{X}\right)\right\}$. 
3. Metric subregularity for generalized equations. Throughout this section, let $X, Y$ be Banach spaces and $F: X \rightrightarrows Y$ be a closed multifunction. Let $b \in Y$ and $a \in F^{-1}(b)$.

Let $J$ denote the normal dual mapping of $Y$, that is,

$$
J(y):=\left\{y^{*} \in \Sigma_{Y^{*}} \mid\left\langle y^{*}, y\right\rangle=\|y\|\right\} \quad \forall y \in Y \backslash\{0\} .
$$

Thus,

$$
J(y)=\partial\|\cdot\|(y) \quad \forall y \in Y \backslash\{0\} .
$$

The following lemma will be useful for our later analysis; it describes quantitatively properties for a point that violates (1.1).

Lemma 3.1. Let $u \in X$ and $\tau, r \in(0,+\infty)$ be such that

$$
\tau d(b, F(u))<r<d\left(u, F^{-1}(b)\right),
$$

and let $\eta, \varepsilon \in(0,+\infty)$. Then there exist $\bar{x} \in X$ and $\bar{y} \in F(\bar{x})$ satisfying the following properties:

$$
\begin{gathered}
\|\bar{x}-u\|<r, \quad 0<\|\bar{y}-b\|<\min \left\{\frac{r}{\tau}, d(b, F(\bar{x}))+\varepsilon\right\}, \\
\|\bar{y}-b\| \leq\|y-b\|+\frac{1}{\tau}(\|x-\bar{x}\|+\eta\|y-\bar{y}\|) \quad \forall(x, y) \in \operatorname{Gr}(F), \\
(0,0) \in\{0\} \times J(\bar{y}-b)+\frac{1}{\tau}\left(B_{X^{*}} \times \eta B_{Y^{*}}\right)+N_{c}(\operatorname{Gr}(F),(\bar{x}, \bar{y})) .
\end{gathered}
$$

If, in addition, $X$ and $Y$ are Asplund spaces, then for any $\sigma>0$ there also exist $\tilde{x}_{\sigma} \in X$ and $y_{\sigma}, \tilde{y}_{\sigma} \in Y \backslash\{b\}$ such that

$$
\max \left\{\left\|\tilde{x}_{\sigma}-\bar{x}\right\|,\left\|\tilde{y}_{\sigma}-\bar{y}\right\|,\left\|y_{\sigma}-\bar{y}\right\|\right\}<\sigma, \quad \tilde{y}_{\sigma} \in F\left(\tilde{x}_{\sigma}\right)
$$

and

$$
(0,0) \in\{0\} \times J\left(y_{\sigma}-b\right)+\frac{1}{\tau}\left(B_{X^{*}} \times \eta B_{Y^{*}}\right)+\hat{N}\left(\operatorname{Gr}(F),\left(\tilde{x}_{\sigma}, \tilde{y}_{\sigma}\right)\right) .
$$

Proof. By (3.1), there exists $\tau^{\prime}>\tau$ such that $\tau^{\prime} d(b, F(u))<r<d\left(u, F^{-1}(b)\right)$. Then there exists $y_{0} \in F(u)$ such that

$$
\left\|y_{0}-b\right\|<\frac{r}{\tau^{\prime}} \quad \text { and } \quad\left(u+r B_{X}\right) \cap F^{-1}(b)=\emptyset .
$$

Define the function $\phi$ by $\phi(x, y):=\|y-b\|$ for all $(x, y) \in X \times Y$. Then $\phi+\delta_{\operatorname{Gr}(F)}$ is lower semicontinuous (due to the closedness of $\operatorname{Gr}(F)$ ), and

$$
\phi\left(u, y_{0}\right)<\frac{r}{\tau^{\prime}} \leq \inf _{(x, y) \in X \times Y}\left(\phi(x, y)+\delta_{\mathrm{Gr}(F)}(x, y)\right)+\frac{r}{\tau^{\prime}} .
$$

Taking $\eta^{\prime} \in(0, \min \{\eta, \tau\})$ with $\frac{2 \eta^{\prime}\left\|y_{0}-b\right\|}{\tau^{\prime}-\eta^{\prime}}<\varepsilon$ and equipping the product $X \times Y$ with the norm

$$
\|(x, y)\|_{\eta^{\prime}}:=\|x\|+\eta^{\prime}\|y\| \quad \forall(x, y) \in X \times Y,
$$

it follows from the Ekeland variational principle that there exists $(\bar{x}, \bar{y}) \in \operatorname{Gr}(F)$ such that

$$
\phi(\bar{x}, \bar{y}) \leq \phi\left(u, y_{0}\right), \quad\left\|(\bar{x}, \bar{y})-\left(u, y_{0}\right)\right\|_{\eta^{\prime}}<r
$$


and

$$
\phi(\bar{x}, \bar{y}) \leq \phi(x, y)+\delta_{\operatorname{Gr}(F)}(x, y)+\frac{1}{\tau^{\prime}}\|(x, y)-(\bar{x}, \bar{y})\|_{\eta^{\prime}} \quad \forall(x, y) \in X \times Y .
$$

Hence

$$
\|\bar{y}-b\| \leq\|y-b\|+\frac{1}{\tau^{\prime}}\left(\|x-\bar{x}\|+\eta^{\prime}\|y-\bar{y}\|\right) \quad \forall(x, y) \in \operatorname{Gr}(F) .
$$

Since $\tau^{\prime}>\tau$ and $\eta>\eta^{\prime}$, this entails (3.3). Substituting $(x, y)$ by $(\bar{x}, y)$ in (3.9), one has

$$
\|\bar{y}-b\| \leq\|y-b\|+\frac{\eta^{\prime}}{\tau^{\prime}}\|y-\bar{y}\| \leq\left(1+\frac{\eta^{\prime}}{\tau^{\prime}}\right)\|y-b\|+\frac{\eta^{\prime}}{\tau^{\prime}}\|\bar{y}-b\| \quad \forall y \in F(\bar{x}) .
$$

This implies that

$$
\|\bar{y}-b\| \leq \frac{\tau^{\prime}+\eta^{\prime}}{\tau^{\prime}-\eta^{\prime}} d(b, F(\bar{x}))=d(b, F(\bar{x}))+\frac{2 \eta^{\prime}}{\tau^{\prime}-\eta^{\prime}} d(b, F(\bar{x})) .
$$

Since $d(b, F(\bar{x})) \leq\|\bar{y}-b\| \leq\left\|y_{0}-b\right\|<\frac{\left(\tau^{\prime}-\eta^{\prime}\right) \varepsilon}{2 \eta^{\prime}}$ (by the first inequality of (3.8) and our choice of $\eta^{\prime}$ ), it follows from (3.7) and (3.8) that (3.2) holds. Let

$$
\phi_{1}(x, y):=\|y-b\|+\frac{1}{\tau^{\prime}}\left(\|x-\bar{x}\|+\eta^{\prime}\|y-\bar{y}\|\right) \quad \forall(x, y) \in X \times Y .
$$

Then (3.9) implies that $(\bar{x}, \bar{y})$ is a minimizer of $\phi_{1}+\delta_{\operatorname{Gr}(F)}$ over $X \times Y$. Noting that $\bar{y} \neq b$ (by (3.2)), it follows from Lemma 2.1 that

$$
\begin{aligned}
(0,0) & \in \partial_{c} \phi_{1}(\bar{x}, \bar{y})+\partial_{c} \delta_{\operatorname{Gr}(F)}(\bar{x}, \bar{y}) \\
& =\{0\} \times J(\bar{y}-b)+\frac{1}{\tau^{\prime}}\left(B_{X^{*}} \times \eta^{\prime} B_{Y^{*}}\right)+N_{c}(\operatorname{Gr}(F),(\bar{x}, \bar{y})) \\
& \subset\{0\} \times J(\bar{y}-b)+\frac{1}{\tau}\left(B_{X^{*}} \times \eta B_{Y^{*}}\right)+N_{c}(\operatorname{Gr}(F),(\bar{x}, \bar{y})) .
\end{aligned}
$$

Hence (3.4) holds. It remains to show the assertion for the case when $X$ and $Y$ are Asplund spaces. In this case, we apply the Asplund space assertion of Lemma 2.1 and note that $\bar{y} \neq b$; thus for any $\sigma>0$ there exist $x_{\sigma}, \tilde{x}_{\sigma} \in X$ and $y_{\sigma}, \tilde{y}_{\sigma} \in Y \backslash\{b\}$ such that (3.5) holds and

$$
(0,0) \in \hat{\partial} \phi_{1}\left(x_{\sigma}, y_{\sigma}\right)+\hat{\partial} \delta_{\mathrm{Gr}(F)}\left(\tilde{x}_{\sigma}, \tilde{y}_{\sigma}\right)+\left(\frac{1}{\tau}-\frac{1}{\tau^{\prime}}\right) B_{(X \times Y)^{*}} .
$$

Noting

$$
\hat{\partial} \phi_{1}\left(x_{\sigma}, y_{\sigma}\right) \subset\{0\} \times J\left(y_{\sigma}-b\right)+\frac{1}{\tau^{\prime}}\left(B_{X^{*}} \times \eta^{\prime} B_{Y^{*}}\right) \text { and } B_{(X \times Y)^{*}}=B_{X}^{*} \times \eta^{\prime} B_{Y^{*}},
$$

it follows that (3.6) holds.

The following theorem (which is motivated by equality (1.3) regarding $\operatorname{reg} F(a, b)$ ) provides an estimate of $\operatorname{subreg} F(a \mid b)$.

Theorem 3.1. Let $X$ and $Y$ be Asplund spaces. Then

$$
\operatorname{subreg} F(a, b) \leq \inf _{\varepsilon>0} \sup \left\{\left\|\hat{D}^{*} F(x, y)^{-1}\right\| \mid x \in B(a, \varepsilon) \backslash F^{-1}(b), y \in F(x) \cap B(b, \varepsilon)\right\} .
$$


Proof. Let $\bar{\tau}$ denote the right-hand side of the above inequality. Suppose the asserted inequality is not true. Then there exists $\tau \in \mathbb{R}$ such that

$$
\bar{\tau}<\tau<\operatorname{subreg} F(a \mid b) .
$$

Then, by (1.4), there exist sequences $\left\{u_{n}\right\}$ in $X \backslash F^{-1}(b)$ and $\left\{r_{n}\right\}$ such that

$$
\left\|u_{n}-a\right\| \rightarrow 0 \text { and } d\left(u_{n}, F^{-1}(b)\right)>r_{n}>\tau d\left(b, F\left(u_{n}\right)\right) \forall n \in \mathbb{N},
$$

where $\mathbb{N}$ denotes the set of all natural numbers. By the Asplund space version of Lemma 3.1 (applied to $u=u_{n}, r=r_{n}, \eta=r_{n}$, and $\sigma=d\left(u_{n}, F^{-1}(b)\right)-r_{n}$ ), there exist $\tilde{x}_{n} \in X$ and $y_{n}, \tilde{y}_{n} \in Y \backslash\{b\}$ such that $\tilde{y}_{n} \in F\left(\tilde{x}_{n}\right)$,

$$
\left\|\tilde{x}_{n}-u_{n}\right\|<r_{n}+\sigma=d\left(u_{n}, F^{-1}(b)\right),\left\|\tilde{y}_{n}-b\right\|<\frac{r_{n}}{\tau}+d\left(u_{n}, F^{-1}(b)\right),
$$

and

$$
(0,0) \in\{0\} \times J\left(y_{n}-b\right)+\frac{1}{\tau}\left(B_{X^{*}} \times r_{n} B_{Y^{*}}\right)+\hat{N}\left(\operatorname{Gr}(F),\left(\tilde{x}_{n}, \tilde{y}_{n}\right)\right) .
$$

Noting that $\hat{N}\left(\operatorname{Gr}(F),\left(\tilde{x}_{n}, \tilde{y}_{n}\right)\right)$ is a cone, it follows that there exist $y_{n}^{*} \in J\left(y_{n}-b\right) \subset$ $\Sigma_{Y^{*}}, u_{n}^{*} \in B_{X^{*}}$, and $v_{n}^{*} \in B_{Y^{*}}$ such that

$$
\left(u_{n}^{*},-\tau y_{n}^{*}-r_{n} v_{n}^{*}\right) \in \hat{N}\left(\operatorname{Gr}(F),\left(\tilde{x}_{n}, \tilde{y}_{n}\right)\right) .
$$

Hence

$$
\tau y_{n}^{*}+r_{n} v_{n}^{*} \in \hat{D}^{*} F\left(\tilde{x}_{n}, \tilde{y}_{n}\right)^{-1}\left(u_{n}^{*}\right) \subset \hat{D}^{*} F\left(\tilde{x}_{n}, \tilde{y}_{n}\right)^{-1}\left(B_{X^{*}}\right) .
$$

This implies that

$$
\left\|\hat{D}^{*} F\left(\tilde{x}_{n}, \tilde{y}_{n}\right)^{-1}\right\| \geq\left\|\tau y_{n}^{*}+r_{n} v_{n}^{*}\right\| \geq \tau-r_{n} .
$$

Noting that $0<r_{n}<d\left(u_{n}, F^{-1}(b)\right) \leq\left\|u_{n}-a\right\| \rightarrow 0$, (3.10) implies that $\left(\tilde{x}_{n}, \tilde{y}_{n}\right) \rightarrow$ $(a, b)$ and $\tilde{x}_{n} \notin F^{-1}(b)$. It follows from (3.11) that $\bar{\tau} \geq \tau$, contradicting our choice of $\tau$.

It is possible that the inequality in Theorem 3.1 is strict. For example, let $X=Y$ and $a, b \in X$ with $a \neq b$. Let $F(a)=\{a, b\}$ and $F(x)=x \forall x \in X \backslash\{a\}$. Then $F$ is closed, and $F^{-1}(b)=\{a, b\}$. For any $\tau \in(0,+\infty)$, let $\delta_{\tau}:=\min \{\tau, 1\} \frac{\|b-a\|}{2}$. Then

$$
d\left(x, F^{-1}(b)\right)=\|x-a\| \leq \frac{\tau\|b-a\|}{2} \leq \tau\|b-x\|=\tau d(b, F(x)) \quad \forall x \in B\left(a, \delta_{\tau}\right) \backslash\{a\} .
$$

It follows from (1.4) that $\operatorname{subreg} F(a, b)=0$. On the other hand, it is easy to verify that

$$
\hat{D}^{*} F(x, y)\left(y^{*}\right)=y^{*} \quad \forall(x, y) \in \operatorname{Gr}(F) \cap((X \backslash\{a\}) \times Y) \text { and } \forall y^{*} \in Y^{*} .
$$

Hence

$$
\inf _{\varepsilon>0} \sup \left\{\left\|\hat{D}^{*} F(x, y)^{-1}\right\| \mid x \in B(a, \varepsilon) \backslash F^{-1}(b), y \in F(x) \cap B(b, \varepsilon)\right\}=1 .
$$

For any $\varepsilon>0$, let

$$
J_{\varepsilon}(y):=\left\{y^{*} \in \Sigma_{Y^{*}} \mid d\left(y^{*}, J(y)\right)<\varepsilon\right\} \quad \forall y \in Y \backslash\{0\} .
$$


For $x \in X$, let $P_{F(x)}(b)$ and $P_{F(x)}^{\varepsilon}(b)$ denote, respectively, the projection and $\varepsilon$ projection of $b$ to $F(x)$, that is,

$$
P_{F(x)}(b):=\{y \in F(x) \mid\|b-y\|=d(b, F(x))\}
$$

and

$$
P_{F(x)}^{\varepsilon}(b):=\{y \in F(x) \mid\|y-b\|<d(b, F(x))+\varepsilon\} \quad \forall x \in X .
$$

Theorem 3.2. Let $\varepsilon, \kappa, \delta \in(0,+\infty)$ be such that

$$
d\left(0, D_{c}^{*} F(x, y)\left(J_{\varepsilon}(y-b)\right)\right) \geq \kappa
$$

$\forall x \in B(a, \delta) \backslash F^{-1}(b)$ and $\forall y \in P_{F(x)}^{\varepsilon}(b) \cap B(b, \delta)$. Then

$$
d\left(x, F^{-1}(b)\right) \leq \frac{1}{\kappa} d(b, F(x)) \quad \forall x \in B\left(a, \frac{\delta}{2+\kappa}\right) .
$$

Proof. Suppose that the conclusion does not hold. Then there exist $u \in B\left(a, \frac{\delta}{2+\kappa}\right)$, $\tau \in\left(\frac{1}{\kappa},+\infty\right)$, and $r \in \mathbb{R}$ such that

$$
\|u-a\| \geq d\left(u, F^{-1}(b)\right)>r>\tau d(b, F(u))
$$

thus

$$
r+\frac{\delta}{2+\delta}<\|u-a\|+\frac{\delta}{2+\delta}<\frac{\delta}{2+\kappa}+\frac{\delta}{2+\delta}<\delta \text { and } \frac{r}{\tau}<\frac{\kappa \delta}{2+\kappa}<\delta .
$$

Take $\eta \in(0, \tau)$ small enough such that

$$
\frac{1}{\tau-\eta}<\kappa \quad \text { and } \quad \min \left\{\frac{2 \eta r}{\tau^{2}}, \frac{2 \eta}{\tau-\eta}\right\}<\varepsilon .
$$

Then, by Lemma 3.1, there exist $\bar{x} \in X$ and $\bar{y} \in F(\bar{x})$ such that (3.2) and (3.4) hold. From $(3.13),(3.14)$, and $(3.2)$, it is easy to verify that

$$
\bar{x} \in B(a, \delta) \backslash F^{-1}(b) \quad \text { and } \quad \bar{y} \in P_{F(\bar{x})}^{\varepsilon}(b) \cap B(b, \delta) .
$$

By (3.4), there exist $y^{*} \in J(\bar{y}-b) \subset \Sigma_{Y^{*}}, x^{*} \in B_{X^{*}}$, and $v^{*} \in B_{Y^{*}}$ such that

$$
\left(\frac{x^{*}}{\tau},-y^{*}-\frac{\eta v^{*}}{\tau}\right) \in N_{c}(\operatorname{Gr}(F),(\bar{x}, \bar{y})) .
$$

Let $\tilde{y}^{*}:=\frac{y^{*}+\frac{\eta v^{*}}{\tau}}{\left\|y^{*}+\frac{\eta v^{*}}{\tau}\right\|}$ and $\tilde{x}^{*}:=\frac{x^{*}}{\tau\left\|y^{*}+\frac{\eta v^{*}}{\tau}\right\|}$. Then, $\tilde{x}^{*} \in D_{c}^{*} F(\bar{x}, \bar{y})\left(\tilde{y}^{*}\right)$, and it follows from (3.15) that $\left\|\tilde{x}^{*}\right\| \leq \frac{1}{\tau\left(1-\frac{n}{\tau}\right)}=\frac{1}{\tau-\eta}<\kappa$ and

$$
\begin{aligned}
\left\|\tilde{y}^{*}-y^{*}\right\| & \leq \frac{\left\|\left(1-\left\|y^{*}+\frac{\eta v^{*}}{\tau}\right\|\right) y^{*}\right\|}{\left\|y^{*}+\frac{\eta v^{*}}{\tau}\right\|}+\frac{\eta\left\|v^{*}\right\|}{\tau\left\|y^{*}+\frac{\eta v^{*}}{\tau}\right\|} \\
& \leq \frac{\frac{\eta}{\tau}}{1-\frac{\eta}{\tau}}+\frac{\eta}{\tau-\eta} \\
& =\frac{2 \eta}{\tau-\eta}<\varepsilon
\end{aligned}
$$


Hence, $\tilde{y}^{*} \in J_{\varepsilon}(\bar{y}-b)$ and $d\left(0, D_{c}^{*}(\bar{x}, \bar{y})\left(J_{\varepsilon}(\bar{y}-b)\right)\right) \leq\left\|\tilde{x}^{*}\right\|<\kappa$. This contradicts (3.12) and (3.16). The proof is completed.

Letting $\delta \rightarrow+\infty$ in Theorem 3.2, we have the following global metric subregularity result.

Corollary 3.1. Suppose that there exist $\varepsilon, \kappa \in(0,+\infty)$ such that

$$
d\left(0, D_{c}^{*} F(x, y)\left(J_{\varepsilon}(y-b)\right)\right) \geq \kappa \quad \forall x \in X \backslash F^{-1}(b) \text { and } \forall y \in P_{F(x)}^{\varepsilon}(b) .
$$

Then

$$
d\left(x, F^{-1}(b)\right) \leq \frac{1}{\kappa} d(b, F(x)) \quad \forall x \in X .
$$

The following example shows that the converses of Theorem 3.2 and Corollary 3.1 do not hold.

Example 3.1. Let $X=Y=R$ and $a=b=0$, and let $F: X \rightarrow 2^{Y}$ be such that $F(0)=[0,+\infty)$ and $F(x)=[1,+\infty)$ for $x \in X \backslash\{0\}$. It is clear that $F$ is closed and that the corresponding (GE) is metrically subregular at $a$. But, by the definition of $F$, it is easy to verify that

$$
N(\operatorname{Gr}(F),(x, 1))=\hat{N}(\operatorname{Gr}(F),(x, 1))=\{0\} \times(-\infty, 0] \quad \forall x \neq a,
$$

and so

$$
N_{c}(\operatorname{Gr}(F),(x, 1))=\{0\} \times(-\infty, 0] \quad \forall x \neq a
$$

(by (2.2)). Hence, for any $\varepsilon \in[0,1)$ and $x \in X \backslash\{a\}$,

$$
D_{c}^{*} F(x, 1)\left(J_{\varepsilon}(1-b)\right)=D^{*} F(x, 1)\left(J_{\varepsilon}(1-b)\right)=D^{*} F(x, 1)(1)=0 .
$$

As a partial converse of Theorem 3.2, we have the following necessity result for (GE) to be metrically subregular.

THEOREM 3.3. Suppose that $F$ is convex and that $(G E)$ is metrically subregular at a. Then there exist $\delta>0$ and a decreasing function $\kappa:[0,1) \rightarrow(0,+\infty)$ such that

$$
d\left(0, D^{*} F(x, y)\left(J_{\varepsilon}(y-b)\right)\right) \geq \kappa(\varepsilon)
$$

$\forall \varepsilon \in[0,1), x \in B(a, \delta) \backslash F^{-1}(b)$, and $y \in F(x)$.

Proof. Since $F$ is metrically subregular at $(a, b)$, there exist $\tau, \delta \in(0,+\infty)$ such that

$$
d\left(x, F^{-1}(b)\right) \leq \tau d(b, F(x)) \quad \forall x \in B(a, \delta) .
$$

Let $x \in B(a, \delta) \backslash F^{-1}(b), y \in F(x)$, and $y^{*} \in J_{\varepsilon}(y-b)$, and let $x^{*} \in D^{*} F(x, y)\left(y^{*}\right)$. Then

$$
\left\langle x^{*}, u-x\right\rangle \leq\left\langle y^{*}, b-y\right\rangle \quad \forall u \in F^{-1}(b),
$$

and there exist $y_{1}^{*} \in J(y-b)$ and $y_{2}^{*} \in \varepsilon B_{Y^{*}}$ such that $y^{*}=y_{1}^{*}+y_{2}^{*}$. It follows that

$$
\left\langle x^{*}, u-x\right\rangle \leq-\left\langle y_{1}^{*}, y-b\right\rangle+\left\langle y_{2}^{*}, b-y\right\rangle \leq-(1-\varepsilon)\|y-b\| \quad \forall u \in F^{-1}(b) .
$$

By (3.18), one has

$$
\left\langle x^{*}, x-u\right\rangle \geq \frac{1-\varepsilon}{\tau} d\left(x, F^{-1}(b)\right) \quad \forall u \in F^{-1}(b) .
$$


This implies that $\left\|x^{*}\right\| \geq \frac{1-\varepsilon}{\tau}$ and hence that $d\left(0, D^{*} F(x, y)(J(y-b))\right) \geq \frac{1-\varepsilon}{\tau}$. Thus (3.17) holds with $\kappa(\varepsilon)=\frac{1-\varepsilon}{\tau}$.

Under some mild restrictions, the coderivative $D_{c}^{*} F(x, y)$ in Theorem 3.2 can be replaced with $\hat{D}^{*} F(x, y)$, as the following result shows.

TheOrem 3.4. Let $X$ be an Asplund space and $Y$ be a Hilbert space. Let $\varepsilon, \kappa, \delta \in$ $(0,+\infty)$ be such that

$$
d\left(0, \hat{D}^{*} F(x, y)\left(J_{\varepsilon}(y-b)\right)\right) \geq \kappa
$$

$\forall x \in B(a, \delta) \backslash F^{-1}(b)$ and $\forall y \in P_{F(x)}^{\varepsilon}(b) \cap B(b, \delta)$. Then

$$
d\left(x, F^{-1}(b)\right) \leq \frac{1}{\kappa} d(b, F(x)) \quad \forall x \in B\left(a, \frac{\delta}{2+\kappa}\right) .
$$

Proof. Suppose that the conclusion does not hold. As at the beginning of the proof of Theorem 3.2, one can take $u \in B\left(a, \frac{\delta}{2+\kappa}\right), \tau \in\left(\frac{1}{\kappa},+\infty\right), r \in \mathbb{R}$, and $\eta \in(0, \tau)$ satisfying (3.13)-(3.15), and by Lemma 3.1 there exists $(\bar{x}, \bar{y}) \in X \times Y$ such that (3.2), (3.3), (3.4), and (3.16) hold. Let

$$
\sigma \in\left(0, \min \left\{\delta-\|\bar{x}-a\|, \delta-\|\bar{y}-b\|, d\left(\bar{x}, F^{-1}(b)\right)\right\}\right)
$$

be sufficiently small such that

$$
\frac{1+\eta+\tau}{\tau} \sigma<\varepsilon-\frac{2 \eta r}{\tau^{2}} \quad \text { and } \quad \frac{4 \sigma}{\|\bar{y}-b\|-\sigma}<\varepsilon-\frac{2 \eta}{\tau-\eta} .
$$

Since $X$ and $Y$ are Asplund spaces, the Asplund space assertion of Lemma 3.1 implies that there exist $\tilde{x}_{\sigma} \in X$ and $y_{\sigma}, \tilde{y}_{\sigma} \in Y \backslash\{b\}$ such that (3.5) and (3.6) hold. By (3.3) (applied to $\left(\tilde{x}_{\sigma}, y\right)$ ), we have

$$
\left\|\tilde{y}_{\sigma}-b\right\|-\left\|\bar{y}-\tilde{y}_{\sigma}\right\| \leq\left(1+\frac{\eta}{\tau}\right)\|y-b\|+\frac{1}{\tau}\left\|\tilde{x}_{\sigma}-\bar{x}\right\|+\frac{\eta}{\tau}\|\bar{y}-b\| \quad \forall y \in F\left(\tilde{x}_{\sigma}\right) .
$$

This and (3.5) imply that

$$
\begin{aligned}
\left\|\tilde{y}_{\sigma}-b\right\|-\sigma & <\left(1+\frac{\eta}{\tau}\right) d\left(b, F\left(\tilde{x}_{\sigma}\right)\right)+\frac{\sigma}{\tau}+\frac{\eta}{\tau}\|\bar{y}-b\| \\
& \leq d\left(b, F\left(\tilde{x}_{\sigma}\right)\right)+\frac{\eta}{\tau}\left(\left\|\tilde{y}_{\sigma}-b\right\|+\|\bar{y}-b\|\right)+\frac{\sigma}{\tau} .
\end{aligned}
$$

Since $\frac{\eta}{\tau}\left(\frac{2 r}{\tau}+\sigma\right)+\frac{\sigma}{\tau}+\sigma<\varepsilon$ (by the first inequality of (3.21)) and

$$
\left\|\tilde{y}_{\sigma}-b\right\|+\|\bar{y}-b\| \leq 2\|\bar{y}-b\|+\left\|\tilde{y}_{\sigma}-\bar{y}\right\|<\frac{2 r}{\tau}+\sigma
$$

(by (3.2) and (3.5)), it follows that $\left\|\tilde{y}_{\sigma}-b\right\|<d\left(b, F\left(\tilde{x}_{\sigma}\right)\right)+\varepsilon$. Thus, by (3.5) and (3.20), one has

$$
\tilde{y}_{\sigma} \in P_{F\left(\tilde{x}_{\sigma}\right)}^{\varepsilon}(b) \cap B(b, \delta) \quad \text { and } \quad \tilde{x}_{\sigma} \in B(a, \delta) \backslash F^{-1}(b) .
$$

By (3.6), there exist $y^{*} \in J\left(y_{\sigma}-b\right), x^{*} \in B_{X^{*}}$, and $v^{*} \in B_{Y^{*}}$ such that

$$
\left(\frac{x^{*}}{\tau},-y^{*}-\frac{\eta v^{*}}{\tau}\right) \in \hat{N}\left(\operatorname{Gr}(F),\left(\tilde{x}_{\sigma}, \tilde{y}_{\sigma}\right)\right) .
$$


Let $\tilde{y}^{*}:=\frac{y^{*}+\frac{\eta v^{*}}{\tau}}{\left\|y^{*}+\frac{\eta v^{*}}{\tau}\right\|}$ and $\tilde{x}^{*}:=\frac{x^{*}}{\tau\left\|y^{*}+\frac{\eta v^{*}}{\tau}\right\|}$. Then, $\tilde{x}^{*} \in \hat{D}^{*} F\left(\tilde{x}_{\sigma}, \tilde{y}_{\sigma}\right)\left(\tilde{y}^{*}\right)$. As in the corresponding part of the proof of Theorem 3.2, we have

$$
\left\|\tilde{x}^{*}\right\|<\kappa \text { and }\left\|\tilde{y}^{*}-y^{*}\right\| \leq \frac{2 \eta}{\tau-\eta} .
$$

Hence $d\left(0, \hat{D}^{*}\left(\tilde{x}_{\sigma}, \tilde{y}_{\sigma}\right)\left(\tilde{y}^{*}\right)\right) \leq\left\|\tilde{x}^{*}\right\|<\kappa$. It follows from (3.19) and (3.22) that $\tilde{y}^{*} \notin$ $J_{\varepsilon}\left(\tilde{y}_{\sigma}-b\right)$. Hence $d\left(\tilde{y}^{*}, J\left(\tilde{y}_{\sigma}-b\right)\right) \geq \varepsilon$. Since $Y$ is a Hilbert space,

$$
J\left(\tilde{y}_{\sigma}-b\right)=\left\{\frac{\tilde{y}_{\sigma}-b}{\left\|\tilde{y}_{\sigma}-b\right\|}\right\} \text { and } y^{*}=\frac{y_{\sigma}-b}{\left\|y_{\sigma}-b\right\|} .
$$

Hence

$$
\left\|\frac{y_{\sigma}-b}{\left\|y_{\sigma}-b\right\|}-\frac{\tilde{y}_{\sigma}-b}{\left\|\tilde{y}_{\sigma}-b\right\|}\right\| \geq\left\|\tilde{y}^{*}-\frac{\tilde{y}_{\sigma}-b}{\left\|\tilde{y}_{\sigma}-b\right\|}\right\|-\left\|y^{*}-\tilde{y}^{*}\right\| \geq \varepsilon-\frac{2 \eta}{\tau-\eta} .
$$

This implies that

$$
\frac{2\left\|y_{\sigma}-\tilde{y}_{\sigma}\right\|}{\left\|\tilde{y}_{\sigma}-b\right\|} \geq \varepsilon-\frac{2 \eta}{\tau-\eta}
$$

thanks to the following elementary inequality:

$$
\frac{2\|u-v\|}{\|v\|} \geq\left\|\frac{u}{\|u\|}-\frac{v}{\|v\|}\right\| \quad \forall u, v \in Y \backslash\{0\} .
$$

On the other hand, (3.5) implies that $\left\|y_{\sigma}-\tilde{y}_{\sigma}\right\|<2 \sigma$ and $\left\|\tilde{y}_{\sigma}-b\right\|>\|\bar{y}-b\|-\sigma$, and it follows from (3.23) that

$$
\varepsilon-\frac{2 \eta}{\tau-\eta} \leq \frac{2\left\|y_{\sigma}-\tilde{y}_{\sigma}\right\|}{\left\|\tilde{y}_{\sigma}-b\right\|}<\frac{4 \sigma}{\|\bar{y}-b\|-\sigma},
$$

contradicting (3.21). The proof is completed.

In the proof of Theorem 3.4, we used the fact that

$$
J(y-b)=\left\{\frac{y-b}{\|y-b\|}\right\} \quad \forall y \in Y \backslash\{b\}
$$

when $Y$ is a Hilbert space. $(* *)$ is not true if $Y$ is not a Hilbert space but an Asplund space. We don't know whether or not Theorem 3.4 (as well as Theorem 3.5(i)) holds when $Y$ is an Asplund space (not a Hilbert space).

It is interesting and significant to establish a point-based characterization for the metric subregularity. Mordukhovich [23] established a characterization for the metric regularity in the finite dimensional setting. To deal with the infinite dimensional setting, Mordukhovich and Shao [26, 27] introduced the concepts of mixed coderivative and partial sequential normal compactness. Recall that the mixed coderivative of $F$ at $(\bar{x}, \bar{y}) \in \operatorname{Gr}(F)$ is a multifunction $D_{M}^{*} F(\bar{x}, \bar{y}): Y^{*} \rightrightarrows X^{*}$ defined as follows: $x^{*} \in D_{M}^{*} F(\bar{x}, \bar{y})\left(y^{*}\right)$ if and only if there exist $x_{n} \rightarrow \bar{x}, y_{n} \rightarrow \bar{y}, x_{n}^{*} \rightarrow x^{*}$, and $y_{n}^{*} \stackrel{w^{*}}{\rightarrow} y^{*}$ such that $y_{n} \in F\left(x_{n}\right)$ and $x_{n}^{*} \in \hat{D}^{*} F\left(x_{n}, y_{n}\right)\left(y_{n}^{*}\right)$ for each $n \in \mathbb{N}$. Also recall that $F$ is partially sequentially normally compact (PSNC) at $(\bar{x}, \bar{y}) \in \operatorname{Gr}(F)$ if, for any sequence $\left\{\left(x_{n}, y_{n}, x_{n}^{*}, y_{n}^{*}\right)\right\}$ in $\operatorname{Gr}(F) \times X^{*} \times Y^{*}$ satisfying

$$
\left(x_{n}, y_{n}\right) \rightarrow(\bar{x}, \bar{y}), x_{n}^{*} \in \hat{D}^{*} F\left(x_{n}, y_{n}\right)\left(y_{n}^{*}\right), x_{n}^{*} \stackrel{w^{*}}{\rightarrow} 0, \text { and } y_{n}^{*} \rightarrow 0,
$$


one has $x_{n}^{*} \rightarrow 0$. By virtue of the mixed coderivative and PSNC, Mordukhovich and Shao [27] (also see [24]) proved the following result in Asplund spaces.

THEOREM MS. $F$ is metrically regular at a for $b$ if and only if $F^{-1}$ is PSNC at $(b, a)$ and $D_{M}^{*} F(a, b)^{-1}(0)=\{0\}$.

In the spirit of Theorem MS, we consider a point-based condition for the metric subregularity. Motivated by the outer coderivative introduced by Ioffe and Outrata [15] in Euclidean spaces, we define the mixed outer coderivative of $F$ at $(\bar{x}, \bar{y}) \in \operatorname{Gr}(F)$ to be a multifunction $D_{M>}^{*} F(\bar{x}, \bar{y}): Y^{*} \rightrightarrows X^{*}$ whose graph consists of all pairs $\left(y^{*}, x^{*}\right)$ such that there exists a sequence of quintuples $\left\{\left(\varepsilon_{n}, x_{n}, y_{n}, x_{n}^{*}, y_{n}^{*}\right)\right\}$ such that $\varepsilon_{n} \rightarrow 0^{+},\left(x_{n}, y_{n}\right) \rightarrow(\bar{x}, \bar{y}), x_{n}^{*} \rightarrow x^{*}$, and $y_{n}^{*} \stackrel{w^{*}}{\rightarrow} y^{*}$ with each $x_{n} \notin F^{-1}(\bar{y})$, $y_{n} \in P_{F\left(x_{n}\right)}^{\varepsilon_{n}}(\bar{y}), y_{n}^{*} \in \mathbb{R}_{+} J_{\varepsilon_{n}}\left(y_{n}-\bar{y}\right)$, and $x_{n}^{*} \in \hat{D}^{*} F\left(x_{n}, y_{n}\right)\left(y_{n}^{*}\right)$.

THEOREM 3.5. The following statements hold:

(i) Let $X$ be an Asplund space and $Y$ be a Hilbert space. Suppose that $F^{-1}$ is PSNC at $(a, b)$. Then $(G E)$ is metrically subregular at a whenever $D_{M>}^{*} F(a, b)^{-1}(0)=\{0\}$.

(ii) Suppose that $F$ is convex. Then $D_{M>}^{*} F(a, b)^{-1}(0)=\{0\}$ whenever (GE) is metrically subregular at a.

Proof. To prove (i), by Theorem 3.4, it suffices to show that there exist $\varepsilon, \delta, \kappa \in$ $(0,+\infty)$ such that $(3.19)$ holds. To do this, suppose to the contrary that there exists a sequence of quintuples $\left\{\left(\varepsilon_{n}, x_{n}, y_{n}, x_{n}^{*}, y_{n}^{*}\right)\right\}$ such that $\varepsilon_{n} \rightarrow 0^{+},\left(x_{n}, y_{n}\right) \rightarrow(a, b)$, and $x_{n}^{*} \rightarrow 0$ with each $x_{n} \notin F^{-1}(b), y_{n} \in P_{F\left(x_{n}\right)}^{\varepsilon_{n}}(b), y_{n}^{*} \in J_{\varepsilon_{n}}\left(y_{n}-b\right) \subset \Sigma_{Y^{*}}$, and $x_{n}^{*} \in \hat{D}^{*} F\left(x_{n}, y_{n}\right)\left(y_{n}^{*}\right)$. Since $B_{Y^{*}}$ is (sequentially) weak*-compact, without loss of generality we assume that $y_{n}^{*} \stackrel{w^{*}}{\rightarrow} y^{*} \in Y^{*}$ (pass to a subsequence if necessary). Hence $0 \in D_{M>}^{*} F(a, b)\left(y^{*}\right)$, that is, $y^{*} \in D_{M>}^{*} F(a, b)^{-1}(0)$ and so $y^{*}=0$. By the PSNC assumption, one has $y_{n}^{*} \rightarrow 0$. This contradicts the fact that $\left\|y_{n}^{*}\right\|=1$ for all $n$.

To prove (ii), let $y^{*} \in D_{M>}^{*} F(a, b)^{-1}(0)$, and suppose that (GE) is metrically regular at $a$. We need to show only that $y^{*}=0$. Let $\left\{\left(\varepsilon_{n}, x_{n}, y_{n}, x_{n}^{*}, y_{n}^{*}\right)\right\}$ be a sequence of quintuples such that $\varepsilon_{n} \rightarrow 0^{+},\left(x_{n}, y_{n}\right) \rightarrow(a, b), x_{n}^{*} \rightarrow 0$, and $y_{n}^{*} \stackrel{w^{*}}{\rightarrow} y^{*}$ with each $x_{n} \notin F^{-1}(b), y_{n} \in P_{F\left(x_{n}\right)}^{\varepsilon_{n}}(b), y_{n}^{*} \in \mathbb{R}_{+} J_{\varepsilon_{n}}\left(y_{n}-b\right)$, and $x_{n}^{*} \in \hat{D}^{*} F\left(x_{n}, y_{n}\right)\left(y_{n}^{*}\right)$. Take $t_{n} \in \mathbb{R}_{+}$and $\tilde{y}_{n}^{*} \in J_{\varepsilon_{n}}\left(y_{n}-b\right)$ such that $y_{n}^{*}=t_{n} \tilde{y}_{n}^{*}$. It suffices to show that $t_{n} \rightarrow 0$. Suppose to the contrary that there exists $t>0$ such that $t_{n}>t$ and

$$
\frac{x_{n}^{*}}{t_{n}} \in \hat{D}^{*} F\left(x_{n}, y_{n}\right)\left(\tilde{y}_{n}^{*}\right) \subset \hat{D}^{*} F\left(x_{n}, y_{n}\right)\left(J_{\varepsilon_{n}}\left(y_{n}-b\right)\right)
$$

for infinitely many $n$. By the convexity of $F$, Theorem 3.3 implies that there exists $\kappa_{0}>0$ such that $\left\|\frac{x_{n}^{*}}{t_{n}}\right\| \geq \kappa_{0}$ for infinitely many $n$, contradicting the fact that $x_{n}^{*} \rightarrow 0$. The proof is completed.

Note that $F$ is automatically PSNC at $(a, b)$ when $Y$ is finite dimensional. The following corollary is immediate from Theorem 3.5.

Corollary 3.2. Let $X$ be an Asplund space and $Y$ be finite dimensional. Suppose that $F$ is convex. Then (GE) is metrically subregular at $a$ if and only if $D_{M>}^{*} F(a, b)^{-1}(0)=\{0\}$.

Remark. By Example 3.1, the convexity assumption cannot be dropped in Theorem 3.5(ii) and Corollary 3.2.

It is both natural and useful to provide conditions ensuring that $\varepsilon$ can be replaced by zero in Theorems 3.2 and 3.4. Before the statement of our next result, let us recall that the set-valued mapping $z \mapsto N_{c}(\operatorname{Gr}(F), z)$ is closed (norm-weak* closed, more precisely) if the following implication holds $\forall(x, y) \in \operatorname{Gr}(F),\left(x^{*}, y^{*}\right) \in X^{*} \times Y^{*}$, nets (generalized sequences) $\left\{\left(x_{n}, y_{n}\right)\right\} \subset \operatorname{Gr}(F)$ and $\left\{\left(x_{n}^{*}, y_{n}^{*}\right)\right\} \subset X^{*} \times Y^{*}$ with each 
$\left(x_{n}^{*}, y_{n}^{*}\right) \in N_{c}\left(\operatorname{Gr}(F),\left(x_{n}, y_{n}\right)\right)$,

$$
\left(x_{n}, y_{n}\right) \rightarrow(x, y),\left(x_{n}^{*}, y_{n}^{*}\right) \stackrel{w^{*}}{\rightarrow}\left(x^{*}, y^{*}\right) \Longrightarrow\left(x^{*}, y^{*}\right) \in N_{c}(\operatorname{Gr}(F),(x, y)) .
$$

Theorem 3.6. Consider the following two cases:

(i) The set-valued mapping $z \mapsto N_{c}(\operatorname{Gr}(F), z)$ is closed, and there exist $\kappa, \delta \in$ $(0,+\infty)$ such that

$$
\operatorname{Gr}(F) \cap\left((a, b)+\delta\left(B_{X} \times B_{Y}\right)\right) \text { is compact }
$$

and

$$
d\left(0, D_{c}^{*} F(x, y)(J(y-b))\right) \geq \kappa
$$

$\forall x \in B(a, \delta) \backslash F^{-1}(b)$ and $\forall y \in P_{F(x)}(b) \cap B(b, \delta) ;$

(ii) $X$ and $Y$ are Asplund spaces, and there exist $\kappa, \delta \in(0,+\infty)$ such that (3.25) holds and

$$
d\left(0, D^{*} F(x, y)(J(y-b))\right) \geq \kappa
$$

$\forall x \in B(a, \delta) \backslash F^{-1}(b)$ and $\forall y \in P_{F(x)}(b) \cap B(b, \delta)$.

Then each of (i) and (ii) implies that

$$
d\left(x, F^{-1}(b)\right) \leq \frac{1}{\kappa} d(b, F(x)) \quad \forall x \in B\left(a, \frac{\delta}{2+\kappa}\right) .
$$

Proof. We proceed as in the proof of Theorem 3.2: supposing that the conclusion does not hold, take $u \in B\left(a, \frac{\delta}{2+\kappa}\right), \tau \in\left(\frac{1}{\kappa},+\infty\right)$, and $r \in \mathbb{R}$ satisfying (3.13) and (3.14). By Lemma 3.1 with $\eta=\varepsilon=\frac{1}{n}$, there exist $\bar{x}_{n} \in X, \bar{y}_{n} \in F\left(\bar{x}_{n}\right)$, $y_{n}^{*} \in J\left(\bar{y}_{n}-b\right) \subset \Sigma_{Y^{*}}, x_{n}^{*} \in B_{X^{*}}$, and $v_{n}^{*} \in B_{Y^{*}}$ such that

$$
\begin{gathered}
\left\|\bar{x}_{n}-u\right\|<r,\left\|\bar{y}_{n}-b\right\|<\frac{r}{\tau}, \\
\left(\frac{x_{n}^{*}}{\tau},-y_{n}^{*}-\frac{v_{n}^{*}}{n \tau}\right) \in N_{c}\left(\operatorname{Gr}(F),\left(\bar{x}_{n}, \bar{y}_{n}\right)\right),
\end{gathered}
$$

and

$$
\left\|\bar{y}_{n}-b\right\| \leq\|y-b\|+\frac{1}{\tau}\left(\left\|x-\bar{x}_{n}\right\|+\frac{1}{n}\left\|y-\bar{y}_{n}\right\|\right)+\delta_{\mathrm{Gr}(F)}(x, y) \quad \forall(x, y) \in X \times Y .
$$

It follows from (3.14) and (3.29) that

$$
\left\|\bar{x}_{n}-a\right\| \leq\left\|\bar{x}_{n}-u\right\|+\|u-a\|<r+\frac{\delta}{2+\kappa}<\delta
$$

and $\left\|\bar{y}_{n}-b\right\|<\delta$. Hence $\left\{\left(\bar{x}_{n}, \bar{y}_{n}\right)\right\}$ is a sequence in $\operatorname{Gr}(F) \cap\left((a, b)+\delta\left(B_{X} \times B_{Y}\right)\right)$. By (3.25) and the weak*-compactness of $B_{X^{*}}$ and $B_{Y^{*}}$, we can assume that

$$
\left(\bar{x}_{n}, \bar{y}_{n}\right) \rightarrow(\bar{x}, \bar{y}) \in \operatorname{Gr}(F), x_{n}^{*} \stackrel{w^{*}}{\rightarrow} x^{*} \in B_{X^{*}} \text { and } y_{n}^{*} \stackrel{w^{*}}{\rightarrow} y^{*} \in B_{Y^{*}}
$$

(passing to generalized subsequences if necessary). It follows from (3.29) and (3.31) that

$$
\bar{y} \in F(\bar{x}),\|\bar{x}-u\| \leq r,\|\bar{y}-b\| \leq \frac{r}{\tau}
$$


and

$$
\|\bar{y}-b\| \leq\|y-b\|+\frac{1}{\tau}\|x-\bar{x}\|+\delta_{\operatorname{Gr}(F)}(x, y) \quad \forall(x, y) \in X \times Y
$$

(in particular $\|\bar{y}-b\|=d(b, F(\bar{x}))$ ). Thus, by (3.32), (3.13), and (3.14), one has

$$
\bar{x} \in B(a, \delta) \backslash F^{-1}(b) \quad \text { and } \quad \bar{y} \in B(b, \delta) \cap P_{F(\bar{x})}(b) .
$$

We now split our proof into two cases.

Case (i). By the assumption that $z \mapsto N_{c}(\operatorname{Gr}(F), z)$ is closed, one can pass to the limit in (3.30) to conclude that $\left(\frac{x^{*}}{\tau},-y^{*}\right) \in N_{c}(\operatorname{Gr}(F),(\bar{x}, \bar{y}))$, and so

$$
d\left(0, D_{c}^{*} F(\bar{x}, \bar{y})\left(y^{*}\right)\right) \leq\left\|\frac{x^{*}}{\tau}\right\| \leq \frac{1}{\tau}<\kappa .
$$

It follows from (3.26) and (3.34) that $y^{*} \notin J(\bar{y}-b)$. Hence $\left\langle y^{*}, \bar{y}-b\right\rangle<\|\bar{y}-b\|$ (because $\left.y^{*} \in B_{Y^{*}}\right)$. But since $y_{n}^{*} \in J\left(\bar{y}_{n}-b\right)$, we also have from (3.33) that

$$
\left\langle y^{*}, \bar{y}-b\right\rangle=\lim _{n \rightarrow \infty}\left\langle y_{n}^{*}, \bar{y}_{n}-b\right\rangle=\lim _{n \rightarrow \infty}\left\|\bar{y}_{n}-b\right\|=\|\bar{y}-b\|,
$$

a contradiction.

Case (ii). Since $X$ and $Y$ are Asplund spaces, one can apply the corresponding assertion of Lemma 3.1 (with $\sigma=\frac{1}{n}$ ) to conclude that there exist $\tilde{x}_{n} \in X, \tilde{y}_{n} \in F\left(\tilde{x}_{n}\right)$, $y_{n} \in Y \backslash\{b\}, \hat{y}_{n}^{*} \in J\left(y_{n}-b\right), \hat{x}_{n}^{*} \in B_{X^{*}}$, and $\hat{v}_{n}^{*} \in B_{Y^{*}}$ such that

$$
\max \left\{\left\|\tilde{x}_{n}-\bar{x}_{n}\right\|,\left\|\tilde{y}_{n}-\bar{y}_{n}\right\|,\left\|y_{n}-\bar{y}_{n}\right\|\right\}<\frac{1}{n}
$$

and

$$
\left(\frac{\hat{x}_{n}^{*}}{\tau},-\hat{y}_{n}^{*}-\frac{\hat{v}_{n}^{*}}{n \tau}\right) \in \hat{N}\left(\operatorname{Gr}(F),\left(\tilde{x}_{n}, \tilde{y}_{n}\right)\right)
$$

Thus we have

$$
\left(\tilde{x}_{n}, \tilde{y}_{n}\right) \rightarrow(\bar{x}, \bar{y}) \quad \text { and } \quad y_{n} \rightarrow \bar{y}
$$

(by (3.33)) and assume without loss of generality that $\hat{x}_{n}^{*} \stackrel{w^{*}}{\rightarrow} \hat{x}^{*} \in B_{X^{*}}$ and $\hat{y}_{n}^{*} \stackrel{w^{*}}{\rightarrow}$ $\hat{y}^{*} \in B_{Y^{*}}$. Passing to the limits in the relation $\hat{y}_{n}^{*} \in J\left(y_{n}-b\right)$ and in (3.35), it follows that $\hat{y}^{*} \in J(\bar{y}-b)$ and $\left(\frac{\hat{x}^{*}}{\tau},-\hat{y}^{*}\right) \in N(\operatorname{Gr}(F),(\bar{x}, \bar{y}))$. This implies that

$$
\frac{\hat{x}^{*}}{\tau} \in D^{*} F(\bar{x}, \bar{y})\left(\hat{y}^{*}\right) \subset D^{*} F(\bar{x}, \bar{y})(J(\bar{y}-b)),
$$

and so $d\left(0, D^{*} F(\bar{x}, \bar{y})(J(\bar{y}-b))\right) \leq \frac{1}{\tau}<\kappa$. But, by (3.27) and (3.34), one also has $d\left(0, D^{*} F(\bar{x}, \bar{y})(J(\bar{y}-b))\right) \geq \kappa$, a contradiction. The proof is completed.

Remark. The coderivative $D^{*} F(x, y)(J(y-b))$ in Theorem 3.6(ii) cannot be replaced by $\hat{D}^{*} F(x, y)(J(y-b))$; we don't even know whether the coderivative $D^{*} F(x, y)$ $(J(y-b))$ can be replaced by $\hat{D}^{*} F(x, y)\left(J_{\varepsilon}(y-b)\right)$ for some $\varepsilon \in(0,1)$ in Theorem 3.6(ii), unlike Theorem 3.4, where, thanks to the Hilbert space assumption, we have made use of the fact that $J(y)=\left\{\frac{y}{\|y\|}\right\}$ for $y \in Y \backslash\{0\}$. 
The following corollary is immediate from Case (ii) of Theorem 3.6.

Corollary 3.3. Let $X$ be finite dimensional and $Y$ be a Euclidean space. Suppose there exists $\kappa \in(0,+\infty)$ such that

$$
d\left(0, D^{*} F(x, y)(y-b)\right) \geq \kappa\|y-b\| \quad \forall x \in X \backslash F^{-1}(b) \text { and } \forall y \in P_{F(x)}(b) .
$$

Then

$$
d\left(x, F^{-1}(b)\right) \leq \frac{1}{\kappa} d(b, F(x)) \quad \forall x \in X .
$$

Based on (1.8), one can provide some sufficient conditions of the metric subregularity for (GE) by finding some conditions which imply that the function $x \mapsto$ $d(b, F(x))$ is lower semicontinuous and that $\partial_{c} d(b, F(\cdot))$ can be described by $D_{c}^{*} F(\cdot, \cdot)$. In this direction, Ledyaev and Zhu [19, Lemmas 3.3 and 3.5] provided the following relationship between $\partial d(b, F(\cdot))$ and $D^{*} F(\cdot, \cdot)$.

Lemma LZ. Let $X$ and $Y$ be Banach spaces with Fréchet smooth Lipschitz bump functions and $F$ be a closed upper semicontinuous multifunction between $X$ and $Y$. Let $U \subset X$ be an open set, and suppose that, for any $x \in U \backslash F^{-1}(b)$,

$$
\sigma \leq \lim _{\varepsilon \rightarrow 0^{+}} \inf \left\{d\left(0, D^{*} F\left(x^{\prime}, y^{\prime}\right)\left(\Sigma_{Y^{*}}\right)\right) \mid x^{\prime} \in B(x, \varepsilon), y^{\prime} \in P_{F\left(x^{\prime}\right)}^{\varepsilon}(b)\right\} .
$$

Then $\sigma \leq d(0, \partial d(b, F(\cdot))(\bar{x}))$ for any $x \in U \backslash F^{-1}(b)$. If, in addition, $F$ is compactvalued, then (3.36) can be replaced with

$$
\sigma \leq \inf \left\{d\left(0, D^{*} F(x, y)\left(\Sigma_{Y^{*}}\right)\right) \mid y \in P_{F(x)}(b)\right\} .
$$

Under the convexity and reflexivity assumption, we have the following exact formula for $\partial d(b, F(\cdot))$.

Lemma 3.2. Let $X$ be a Banach space and $Y$ be a reflexive Banach space. Suppose that $F: X \rightrightarrows Y$ is a closed convex multifunction. Then the following statements hold:

(i) For any $x \in \operatorname{dom}(F), P_{F(x)}(b) \neq \emptyset$.

(ii) The function $x \mapsto d(b, F(x))$ is lower semicontinuous.

(iii) For any $x \in \operatorname{dom}(F) \backslash F^{-1}(b)$ and $y \in P_{F(x)}(b)$,

$$
\partial d(b, F(\cdot))(x)=D^{*} F(x, y)(J(y-b)) .
$$

Proof. (i) Let $x \in \operatorname{dom}(F)$. Since $F$ is closed and convex, $F(x)$ is a closed convex nonempty subset of $Y$. It follows from the reflexivity of $Y$ that $P_{F(x)}(b) \neq \emptyset$.

(ii) Suppose to the contrary that $F$ is not lower semicontinuous at some $x_{0} \in X$. Then there exists a sequence $\left\{x_{n}\right\}$ in $X$ such that $x_{n} \rightarrow x_{0}$ and

$$
d\left(b, F\left(x_{0}\right)\right)>\lim _{n \rightarrow \infty} d\left(b, F\left(x_{n}\right)\right) .
$$

By (i), take $y_{n} \in F\left(x_{n}\right)$ such that $\left\|y_{n}-b\right\|=d\left(b, F\left(x_{n}\right)\right)$. By (3.37) and the reflexivity of $Y$, without loss of generality we assume that $y_{n} \stackrel{w}{\rightarrow} y_{0}$. Hence $\left(x_{n}, y_{n}\right) \stackrel{w}{\rightarrow}\left(x_{0}, y_{0}\right)$. It follows from the convexity and closedness of $F$ that $y_{0} \in F\left(x_{0}\right)$. Hence,

$$
d\left(b, F\left(x_{0}\right)\right) \leq\left\|y_{0}-b\right\| \leq \lim _{n \rightarrow \infty}\left\|y_{n}-b\right\|=\lim _{n \rightarrow \infty} d\left(b, F\left(x_{n}\right)\right),
$$

contradicting (3.37). 
(iii) Let $x \in \operatorname{dom}(F) \backslash F^{-1}(b), y \in P_{F(x)}(b)$, and $x^{*} \in \partial d(b, F(\cdot))(x)$. Then

$$
\left\langle x^{*}, u-x\right\rangle \leq d(b, F(u))-d(b, F(x)) \leq\|v-b\|-\|y-b\| \quad \forall(u, v) \in \operatorname{Gr}(F) .
$$

Let $\psi(u, v):=\|v-b\|+\delta_{\operatorname{Gr}(F)}(u, v) \forall(u, v) \in X \times Y$. By (3.38), we have

$$
\left\langle x^{*}, u-x\right\rangle \leq \psi(u, v)-\psi(x, y) \quad \forall(u, v) \in X \times Y .
$$

This means that $\left(x^{*}, 0\right) \in \partial \psi(x, y)=\{0\} \times J(y-b)+N(\operatorname{Gr}(F),(x, y))$, that is, $x^{*} \in D^{*} F(x, y)(J(y-b))$. Hence $\partial d(b, F(\cdot))(x) \subset D^{*} F(x, y)(J(y-b))$. Conversely, let $y^{*} \in J(y-b)$ and $x^{*} \in D^{*} F(x, y)\left(y^{*}\right)$. Then for any $(u, v) \in \operatorname{Gr}(F)$,

$\left\langle x^{*}, u-x\right\rangle \leq\left\langle y^{*}, v-y\right\rangle=\left\langle y^{*}, v-b\right\rangle-\left\langle y^{*}, y-b\right\rangle \leq\|v-b\|-\|y-b\|=\|v-b\|-d(b, F(x))$.

Hence $\left\langle x^{*}, u-x\right\rangle \leq d(b, F(u))-d(b, F(x)) \forall u \in X$. This shows that $x^{*} \in \partial d(b, F(\cdot))(x)$. The proof is completed.

Based on (1.8), Lemma 3.2, and Theorem 3.3, we have the following characterizations of the metric subregularity for a convex closed multifunction.

Proposition 3.1. Let $Y$ be a reflexive Banach space, and suppose that $F$ is convex and closed. Then the following statements are equivalent:

(i) $(G E)$ is metrically subregular at a.

(ii) There exist $\kappa, \delta \in(0,+\infty)$ such that

$$
d\left(0, D^{*} F(x, y)(J(y-b))\right) \geq \kappa \quad \forall x \in B(a, \delta) \backslash F^{-1}(b) \text { and } \forall y \in P_{F(x)}(b) \cap B(b, \delta) .
$$

(iii) There exist $\kappa, \delta \in(0,+\infty)$ such that

$$
d\left(0, D^{*} F(x, y)(J(y-b))\right) \geq \kappa \quad \forall x \in B(a, \delta) \backslash F^{-1}(b) \text { and } \forall y \in F(x) .
$$

Acknowledgments. The authors wish to thank the referees for careful reading of the paper and valuable comments.

\section{REFERENCES}

[1] D. Aze, A unified theory for metric regularity of multifunctions, J. Convex Anal., 13 (2006), pp. $225-252$.

[2] J.F. Bonnans And A. Shapiro, Perturbation Analysis of Optimization Problems, Springer, New York, 2000.

[3] J.V. Burke And S. Deng, Weak sharp minima revisited, Part III: Error bounds for differentiable convex inclusions, Math. Program., 116 (2009), pp. 37-56.

[4] F.H. Clarke, Optimization and Nonsmooth Analysis, Wiley, New York, 1983.

[5] A.L. Dontchev, A.S. Lewis, and R.T. Rockafellar, The radius of metric regularity, Trans. Amer. Math. Soc., 355 (2003), pp. 493-517.

[6] A.L. Dontchev and R.T. Rockafellar, Regularity and conditioning of solution mappings in variational analysis, Set-Valued Anal., 12 (2004), pp. 79-109.

[7] M. Fabian, R. Henrion, A. Kruger, and J.V. Outrata, Error bounds: Necessary and sufficient conditions, Set-Valued and Variational Anal., in press (DOI 10.1007/s11228-0100133-0).

[8] R. Henrion and A. Jourani, Subdifferential conditions for calmness of convex constraints, SIAM J. Optim., 13 (2002), pp. 520-534.

[9] R. Henrion, A. Jourani, and J. Outrata, On the calmness of a class of multifunctions, SIAM J. Optim., 13 (2002), pp. 603-618.

[10] R. Henrion and J. Outrata, Calmness of constraint systems with applications, Math. Program., 104 (2005), pp. 437-464.

[11] H. Hu, Characterizations of the strong basic constraint qualification, Math. Oper. Res., 30 (2005), pp. 956-965. 
[12] H. Hu, Characterizations of local and global error bounds for convex inequalities in Banach spaces, SIAM J. Optim., 18 (2007), pp. 309-321.

[13] A.D. Ioffe, Regular points of Lipschitz functions, Trans. Amer. Math. Soc., 251 (1979), pp. 6169.

[14] A.D. Ioffe, Metric regularity and subdifferential calculus, Russian Math. Surveys, 55 (2000), pp. 501-558.

[15] A.D. Ioffe and J.V. Outrata, On metric and calmness qualification conditions in subdifferential calculus, Set-Valued Anal., 16 (2008), pp. 199-277.

[16] A. Jourani And L. Thibault, Metric regularity and subdifferential calculus in Banach spaces, Set-Valued Anal., 3 (1995), pp. 87-100.

[17] A. Jourani and L. Thibault, Verifiable conditions for openness and metric regularity of multivalued mappings, Trans. Amer. Math. Soc., 347 (1995), pp. 1225-1268.

[18] D. Klatte and B. Kummer, Nonsmooth Equations in Optimization: Regularity, Calculus, Methods and Applications, Nonconvex Optim. Appl., 60 (2002), Kluwer Academic Publishers, Dordrecht.

[19] Y. LedYaev AND Q.J. Zhu, Implicit multifunction theorem, Set-Valued Anal., 7 (1999), pp. 209-238.

[20] A. Lewis AND J.S. PAng, Error bounds for convex inequality systems, in Generalized Convexity, Generalized Monotonicity: Recent Results, Proceedings of the 5th Symposium on Generalized Convexity, Luminy, J.-P. Crouzeix, J.-E. Martinez-Legaz, and M. Volle, eds., Kluwer Academic Publishers, Dordrecht, 1997, pp. 75-100.

[21] W. LI, Abadie's constraint qualification, metric regularity, and error bounds for differentiable convex inequalities, SIAM J. Optim., 7 (1997), pp. 966-978.

[22] W. Li And I. Singer, Global error bounds for convex multifunctions and applications, Math. Oper. Res., 23 (1998), pp. 443-462.

[23] B.S. Mordukhovich, Complete characterization of openness, metric regularity, and Lipschitzian properties of multifunctions, Trans. Amer. Math. Soc., 340 (1993), pp. 1-35.

[24] B.S. Mordukhovich, Variational Analysis and Generalized Differentiation I/II, SpringerVerlag, Berlin, 2006.

[25] B.S. Mordukhovich And Y. ShaO, Nonsmooth sequential analysis in Asplund spaces, Trans. Amer. Math. Soc., 348 (1996), pp. 1235-1280.

[26] B.S. Mordukhovich And Y. ShaO, Nonconvex coderivative calculus for infinite-dimensional multifunction, Set-Valued Anal., 4 (1996), pp. 205-236.

[27] B.S. Mordukhovich And Y. Shao, Stability of multifunctions in infinite dimensions: Point criteria and applications, SIAM J. Control Optim., 35 (1997), pp. 285-314.

[28] K.F. NG AND X.Y. Zheng, Error bounds for lower semicontinuous functions in normed spaces, SIAM J. Optim., 12 (2001), pp. 1-17.

[29] H.V. NGai And M. Thera, Metric inequality, subdifferential calculus and applications, SetValued Anal., 9 (2001), pp. 187-216.

[30] H.V. NGai And M. Thera, Error bounds and implicit multifunctions in smooth Banach spaces and applications to optimization, Set-Valued Anal., 12 (2004), pp. 195-223.

[31] R.R. PhelPs, Convex Functions, Monotone Operators and Differentiability, Lecture Notes in Math. 1364, Springer, New York, 1989.

[32] S.M. Robinson, Regularity and stability for convex multivalued functions, Math. Oper. Res., 1 (1976), pp. 130-143.

[33] J.J. Ye AND X.Y. Ye, Necessary optimality conditions for optimization problems with variational inequality constraints, Math. Oper. Res., 22 (1997), pp. 977-997.

[34] C. Zalinescu, A nonlinear extension of Hoffman's error bounds for linear inequalities, Math. Oper. Res., 28 (2003), pp. 524-532.

[35] X.Y. Zheng And K.F. NG, Metric regularity and constraint qualifications for convex inequalities on Banach spaces, SIAM J. Optim., 14 (2003), pp. 757-772.

[36] X.Y. Zheng AND K.F. NG, Metric subregularity and constraint qualifications for convex generalized equations in Banach spaces, SIAM. J. Optim., 18 (2007), pp. 437-460.

[37] X.Y. Zheng AND K.F. NG, Calmness for L-subsmooth multifunctions in Banach spaces, SIAM J. Optim., 19 (2009), pp. 1648-1673. 